\title{
Testing the Setting and Hardening of Concrete Using the Prism Technique
}

\author{
F. Kemiha, A. Bouhadjera, M. Grimes and T. benkedidah \\ NDT Lab, Abdelhak ben Hamouda University, Jijel, Algeria
}

\begin{abstract}
In non-destructive testing of concrete, the ultrasonic pulse-echo technique has proven to be a reliable method for locating cracks and other internal defects. An electro-mechanical transducer is used to generate a short pulse of ultrasonic stress waves that propagate into the object being inspected. Reflection of the stress pulse occurs at boundaries separating materials with different densities and elastic properties. The reflected pulse travels back to the transducer that also acts as a receiver. The received signal can give many insights to the properties of materials under test. The question arises how the setting and hardening of cement paste, mortar, and concrete can be measured continuously. Continuous measurement asks for non-destructive methods. The current testing method such as the vicat needle for cement paste and penetration resistance test for concrete methods measure at intervals. These methods can be applied before the end of setting. All these methods do not allow continuous measurements and are partly destructive. In this paper a special pulse-echo technique called "prism technique" is used to evaluate the evolution of proprieties of concrete over time. For that, an automatic system has been developed with LabVIEW program in order to monitor the time of flight of the reflected p-wave. Two mixtures of mortar are made using different proportions, of water, cement, and sand. The mortar is poured into a mould that has a form of a prism. Measurements are taken every 10 minutes over a period of 24 hours. This enabled us to plot the evolution of $\mathrm{p}$-wave velocities over time for the samples and compare the results.
\end{abstract}

Key words: Setting of concrete, hardening of concrete, p-wave velocity, prism technique, ultrasonic system.

\section{Introduction}

The exact course of the solidification process or of hardening process of materials such as cement, mortar, and concrete plays a decisive role in deciding whether the fully hardened construction element has reached its planned mechanical properties, in particular its strength, or not. Since the hardening process depends on a number of parameters such us on the capacities of the concrete mixer, on the water-to-cement ratio, on the environmental temperature, etc. The setting of cement has great influence on the available duration of workability of the fresh concrete.

The proposed method is based on a testing technique designed by A. Bouhadjera [1-3]. For convenience, it is called the prism technique, with reference to the characteristic shape of the samples used. It has been applied successfully for the

Corresponding author: F. Kemiha, magister, assistant master, research field: ultrasonic testing. evaluation of elastic properties of isotropic materials such as mortar and concrete.

\section{Theory of Ultrasound}

\subsection{Basic Relationships}

When a disturbance (stress or displacement) is applied suddenly at a point on the surface of a solid, such as by impact or by pulse, the disturbance propagates through the solid as three different types of stress waves: a P-wave, an S-wave, and an R-wave. In an infinite isotropic, elastic solid, the p-wave speed, $\mathrm{C}_{\mathrm{p}}$, is related to the Young's modulus of elasticity, E Poisson's ratiov, and the density, $\rho$ as follows:

$$
C_{p}=\sqrt{\frac{E(1-v)}{\rho(1+v)(1-2 v)}}
$$

The S-wave propagates at a lower speed, Cs, is given by: 


$$
C_{s}=\sqrt{\frac{G}{\rho}}=\sqrt{\frac{E}{2 \rho(1+v)}}
$$

Where $\mathrm{G}=$ the shear modulus of elasticity.

The ratio of S-wave speed to $\mathrm{P}$-wave speed depends on Poisson's ration as follows:

$$
\frac{C_{s}}{C_{p}}=\sqrt{\frac{1-2 v}{2(1-v)}}
$$

For Poisson's ration of 0.2 , which is typical of concrete, this ratio equals 0.61 . The ratio of the $\mathrm{R}$-wave speed $\mathrm{C}_{\mathrm{r}}$, to the $\mathrm{S}$-wave speed is given by the following approximate formula:

$$
\frac{\mathrm{C}_{\mathrm{r}}}{\mathrm{C}_{\mathrm{s}}}=\frac{0.87+1.12 v}{1+v}
$$

For Poisson's ratio equal to 0.2 , the R-wave speed is $92 \%$ of the $\mathrm{S}$-wave speed.

\subsection{Reflection at Interface}

When an ultrasound wave travelling through material 1 is incident on the interface between a dissimilar material 2, a portion of incident wave is reflected (Fig. 1). The amplitude of the reflection is function of the angle of incidence and is a maximum when this angle is $90^{\circ}$ (normal incidence). For normal incidence the reflection coefficient, $\mathrm{R}$ is given by the following formula [5]:

$$
R=\frac{Z_{2}-Z_{1}}{Z_{2}+Z_{1}}
$$

Where:

Z2 = specific acoustic impedance of material 2, and $\mathrm{Z} 1=$ specific acoustic impedance of material 1 .

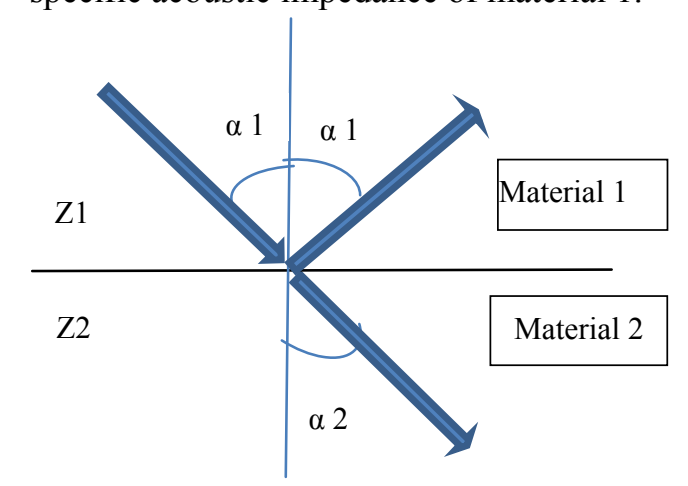

Fig. 1 Incident, refracted, and reflected wave.
The specific impedance is the product of the wave speed and density of the material:

$$
\mathrm{Z}=\mathrm{v} * \rho
$$

The reflection coefficient given by Eq. 5 can be negative or positive depending on the relative values of the acoustic impedances of the two materials. If $\mathrm{Z} 2$ $<Z 1$, such as would occur at a concrete-air interface, the reflection coefficient is negative. This means that the sign of the stress in the reflected wave is opposite to the sign of the stress in the incident wave. Thus an incident P-wave with a compressive stress would reflect as a $\mathrm{P}$-wave with a tensile stress. If $\mathrm{Z} 2>\mathrm{Z} 1$, the reflection coefficient is positive and there is no change in the sign of the stress. In this case, an incident $\mathrm{P}$-wave with compressive stress would reflect back a wave with compressive stress. These differences are important in distinguishing between reflection from a concrete-air interface and from a concrete-steel interface [5].

\section{Prism Technique}

\subsection{The Old Prism Technique}

The old prism technique is used for testing hard material samples. The transducer-cell configuration is represented in Fig. 2. The main parts of the cell are two concentric discs of perplex $5 \mathrm{~mm}$ thick. The one in the centre is fixed to the bottom of the cell, whereas the outside one is made to turn with no play around the fixed disc, making a circle with radius $\mathrm{R}$. The transducer is fixed on the top of the ring at a distance $\mathrm{R}$ from the centre of the disc. The SUT (specimen under test) is centred on the disc with its biggest face opposite to the transducer. The angle $\alpha$ is made to vary between $0^{\circ}$ (normal incidence on the main face of SUT), and $90^{\circ}$ (Transducer beam parallel to the main face) [4].

The velocity of ultrasound waves is evaluated from the following formula:

$$
\mathrm{C}_{\mathrm{p}, \mathrm{s}}=\mathrm{a} /\left(\mathrm{T}_{\mathrm{p}, \mathrm{s}} \mathrm{t}\right)
$$

a: side of the prism;

$\mathrm{T}_{\mathrm{p}, \mathrm{s}}$ : Times of flight of pulse related to both 
compressional and shear waves when refraction takes place at the critical angles.

$\mathrm{t}$ : total time of flight.

Fig.2.b shows a general view of the ultrasonic apparatus. It consists of the transducer cell, an ultrasonic pulser-receiver, a digital oscilloscope and a laptop computer for data acquisition. The transducer frequencies used for measurement is $1 \mathrm{Mhz}$. The prism-shaped specimen could be seen clearly on the right of Fig. 2.b.

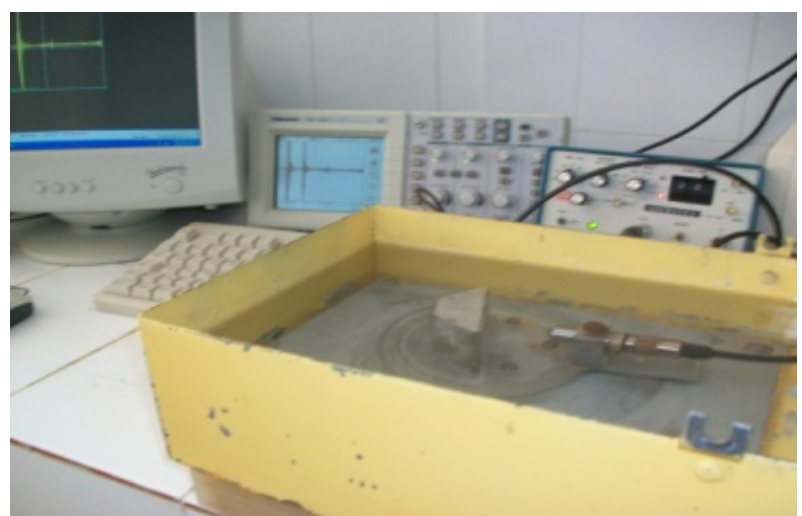

(a)
Figs. 3 and 4 give waveform related to compressional waves and shear waves for a concrete sample obtained with this technique.

The procedure for evaluating time of flights is as follows: At the start of the experiment, the angle $\alpha$ is set to zero. This gives a very clear echo on the oscilloscope related to the total reflection from SUT and represents the time delay of the ultrasound pulse within the water and interface. It enables the evaluation of time of flight of compression wave (first

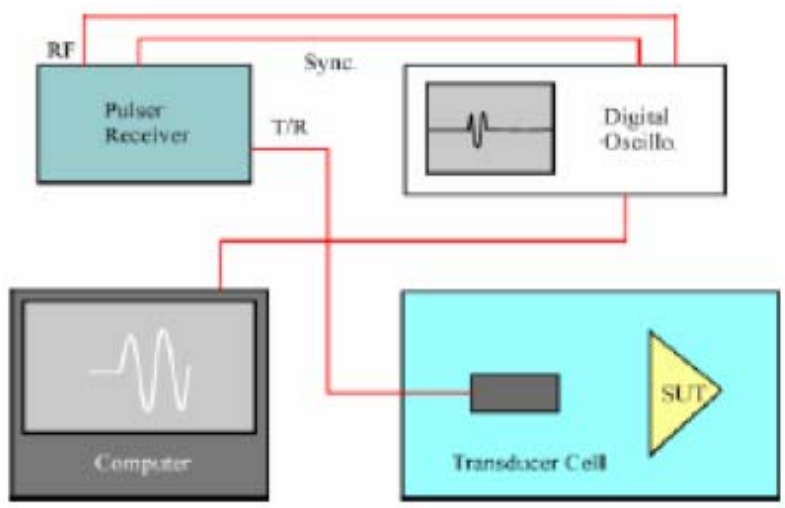

(b)

Fig. 2 a) Main parts of the cell; b) Schematic diagram.

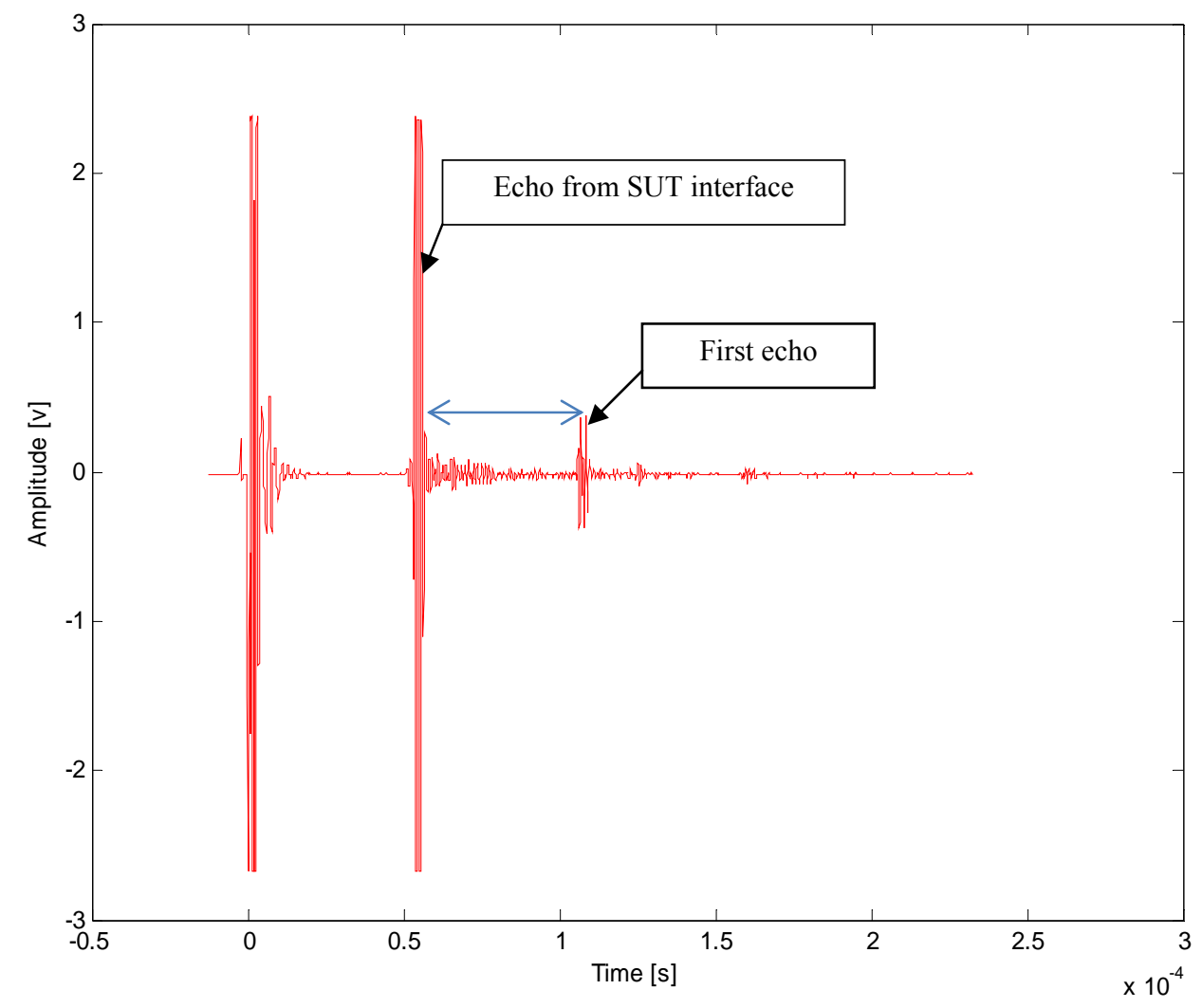

Fig. 3 P-wave. 


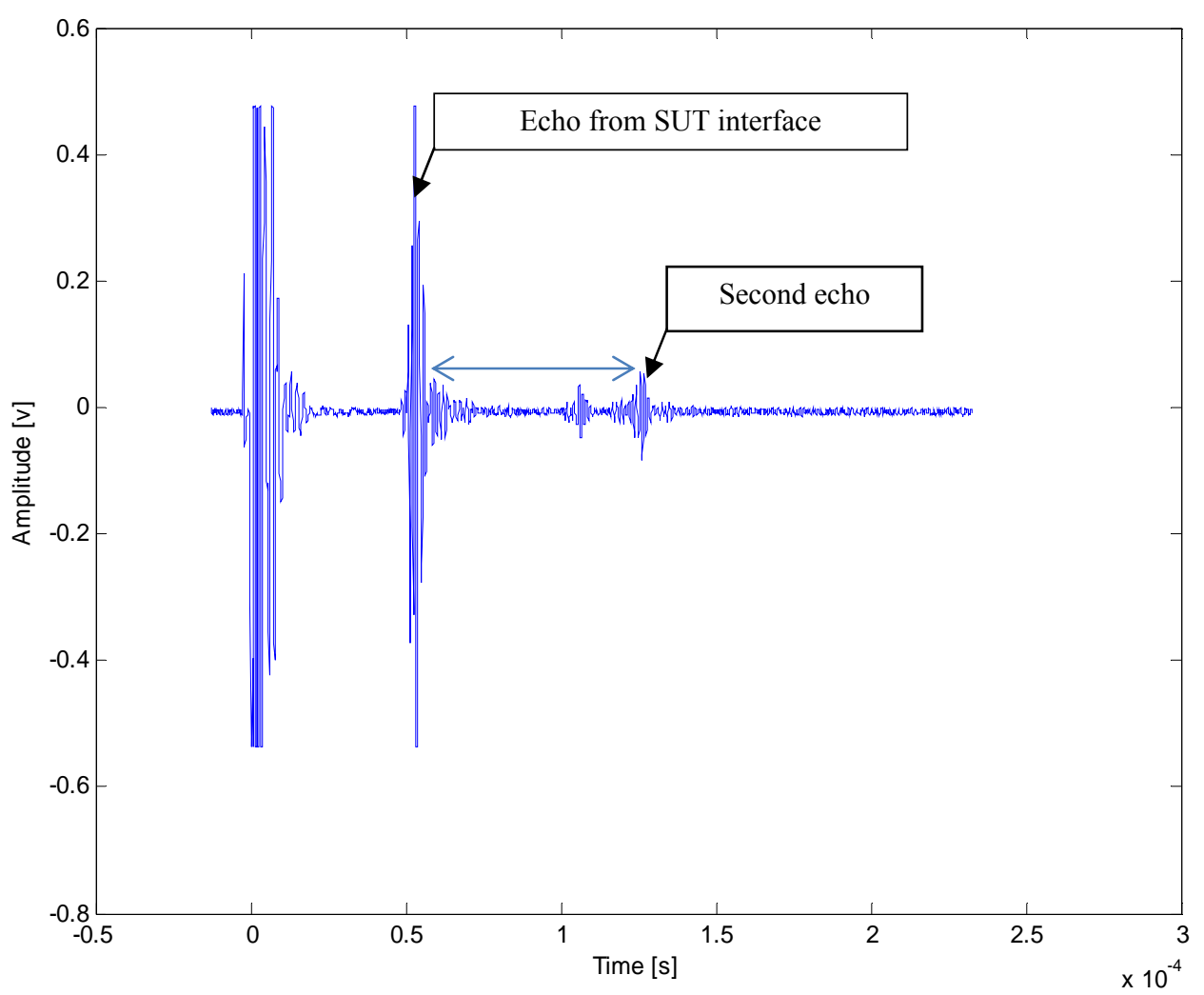

Fig. 4 S-wave.

echo) by using the cursors of the digital oscilloscope. After that, the angle is creased until the appearance of a second echo (Fig. 4), which is related to shear waves. It coincides with the first critical angle which gives rise to a refracted shear wave at an angle of $45^{\circ}$ within SUT. This method can measure an $\mathrm{s}$ and $\mathrm{p}$-velocity of ultrasound wave in hard concrete only. To view the change of this velocity during a setting and hardening a new apparatus has been developed.

\subsection{The New Prism Technique}

The new prism technique is used for testing the setting and hardening of concrete. The principle of the transmission method is show in Figs. 4.a and 5.b. Bouhadjera in 2009 developed an apparatus for non-destructive testing of samples. The samples can be formed from critical materials which change their properties during manufacture, for example on a hardening or solidification. The device requires only one parameter, namely, the TOF (time of flight), to be determined accurately for multiple measurements.
P-wave velocity and s-wave velocity are measured with this technique according to Eq. 6 .

The invention can furthermore be used to characterize other materials which pass through a phase transition or which change as a consequence of other mechanical, thermal, chemical or biological processes [3].

The principle of the transmission method is shown in Fig. 5.c. A pulse is generated at a surface of the specimen and ultrasound wave is travelling through the material and picked up at the other side. The incident pulse and the reflected pulse are recorded, converted to a digital signal and stored in a memory for further or immediate evaluation.

The approach is based on the observation of waves transmitted and reflected through mortar or concrete during setting and hardening. The ultrasound wave is recorded and analyzed during the hardening of the material continuously. That wave form as well as wave parameters like travel time (related to the wave velocity), amplitude (related to the wave energy) and 

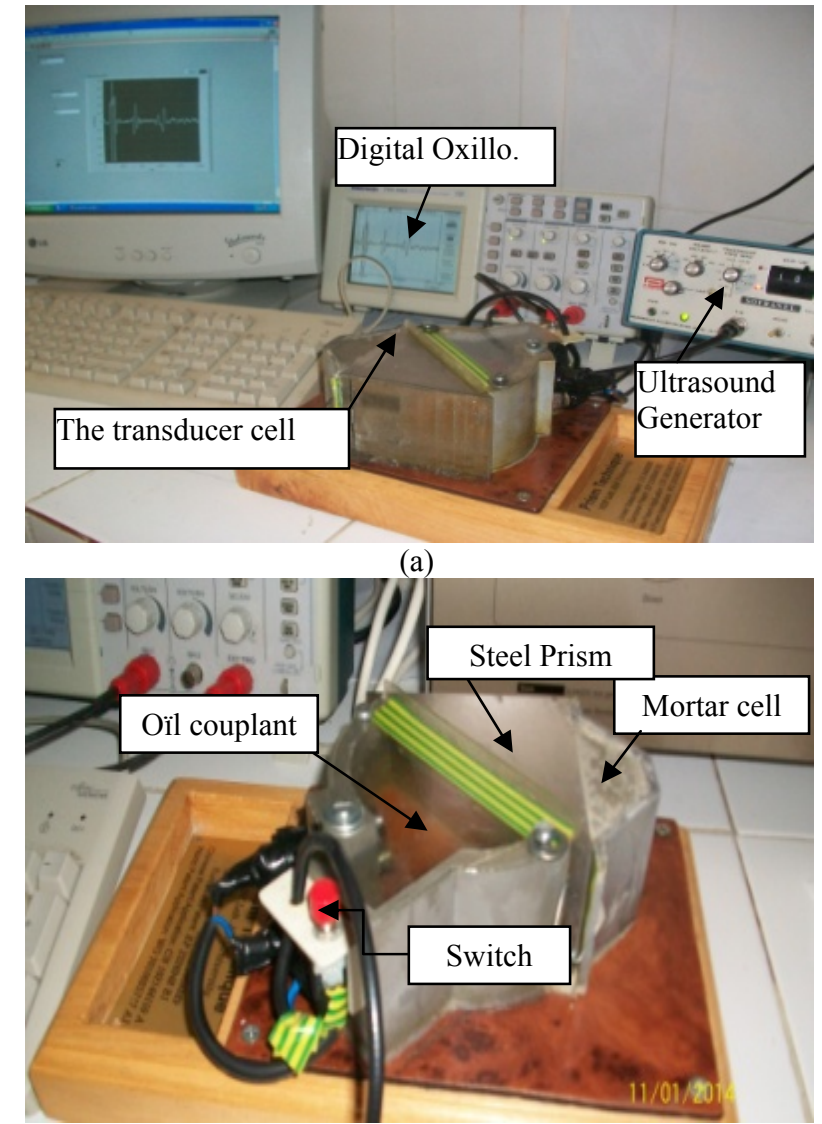

(b)

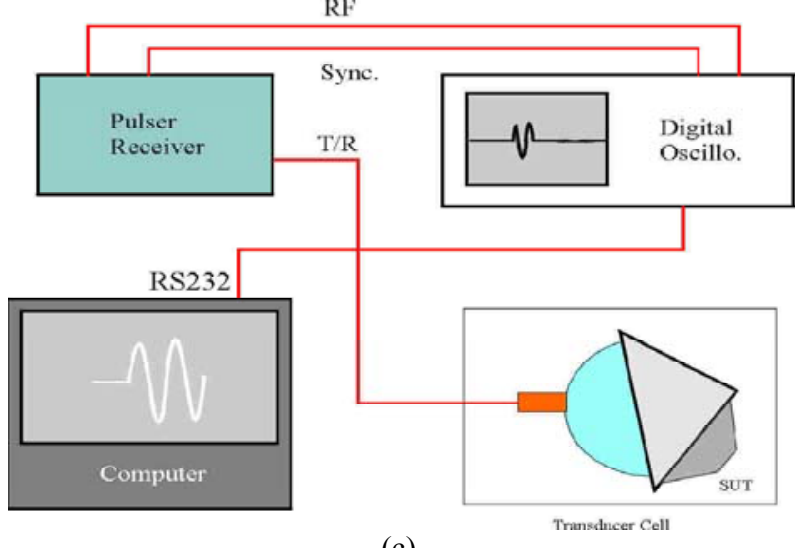

(c)

Fig. 5 a) General view of the new ultrasonic apparatus; b) Apparatus of prism technique; c) Schematic diagram.

frequency content of the signal are influenced by elastic properties of material. Cement based materials like concrete and mortar are changing its status from a suspension to a water sutured porous media during hardening. This change can be observed recording transient waves transmitted through the material. Usually, these transients are recorded in certain intervals to document the changes in signal wave form during setting and hardening more obvious. Fig. 6.a and Fig. 5.b show the ultrasound signal of p-wave and it Fourier transforms. The changes in signal wave form during setting are give in Fig. 6.c.

\subsection{Wavelet Transforms}

The wavelet transformation is a mathematical procedure for the evaluation of signals. Unlike to the well-known Fourier transform the wavelet transformed signal shows the frequency domain and time domain. In order to gather as much information as possible from the raw signal to evaluate concrete and mortar.

The wavelet transformed signal shows the frequency domain and the time information is usually hidden in the imaginary part of the transform, using wavelet, the time information can be obtain mush clearer. Fig. 7 shows the wavelet transformed of ultrasound signal obtained after transmitting fresh concrete [6].

\section{Results Experimental}

The time of flight of ultrasonic wave has been calculated from measurement and stored from different hours. Table 1 shows some preliminary results related to compression velocity, and young's modulus of two samples of concrete with w/c ratios varying from 0.3 to 0.6 .

The p-wave velocity is calculated from the time of flight of p-wave measurement. Figs. 8 and 9 show all results for the concrete composition according to Table 1. The graph shows the known phenomenon. The p-wave changes the $500 \mathrm{~m} / \mathrm{s}$ to $3,000 \mathrm{~m} / \mathrm{s}$ afters 24 hours. For fresh cement, the evolutions of $c p(t)$ in relation to time are characterized by:

- An initial velocity of the order of $500 \mathrm{~m} / \mathrm{s}$;

- A strong evolution during the setting process;

- An initial period with slow evolution, but for which the device is able to detect a slight mechanical evolution;

- A noticeable transition time (ts) between two very different evolution periods: this time is sensitive 
to changes in material composition.

The $\mathrm{p}$-wave velocity is sensitive to the modification of compressive properties.

The effect of the ratio $\mathrm{E} / \mathrm{C}$ is also seen in the graphs of Figs.8 and 9. The increases of this ratio go to a change of velocity.
The evolution of young's modulus, reflection energy, and reflections from the two samples are shown in Figs. 10 and 11.

With increasing hardening time the reflection become larger since the test material gets harder. Results are in good agreement with these obtained in Refs. [6-8].

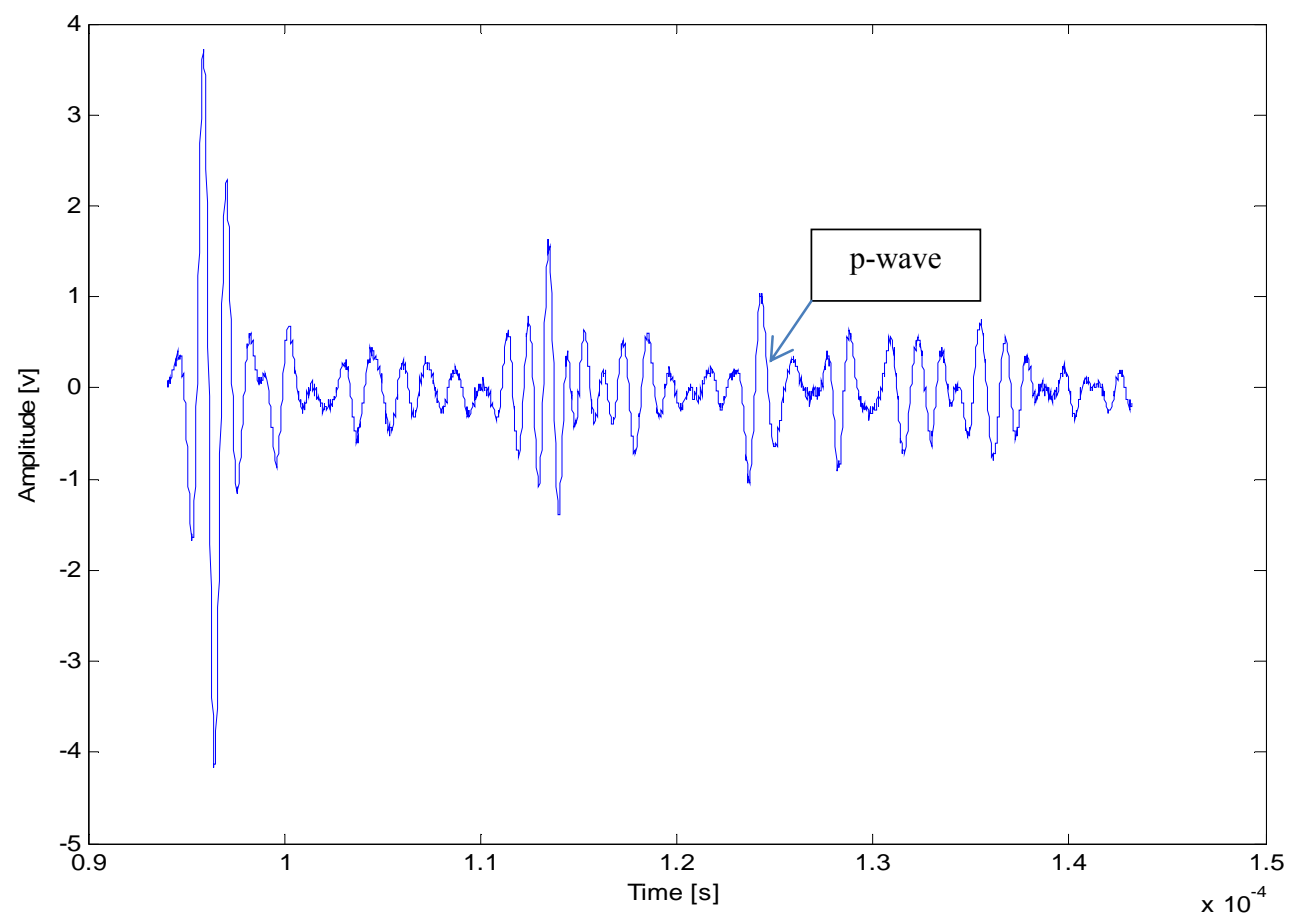

(a)

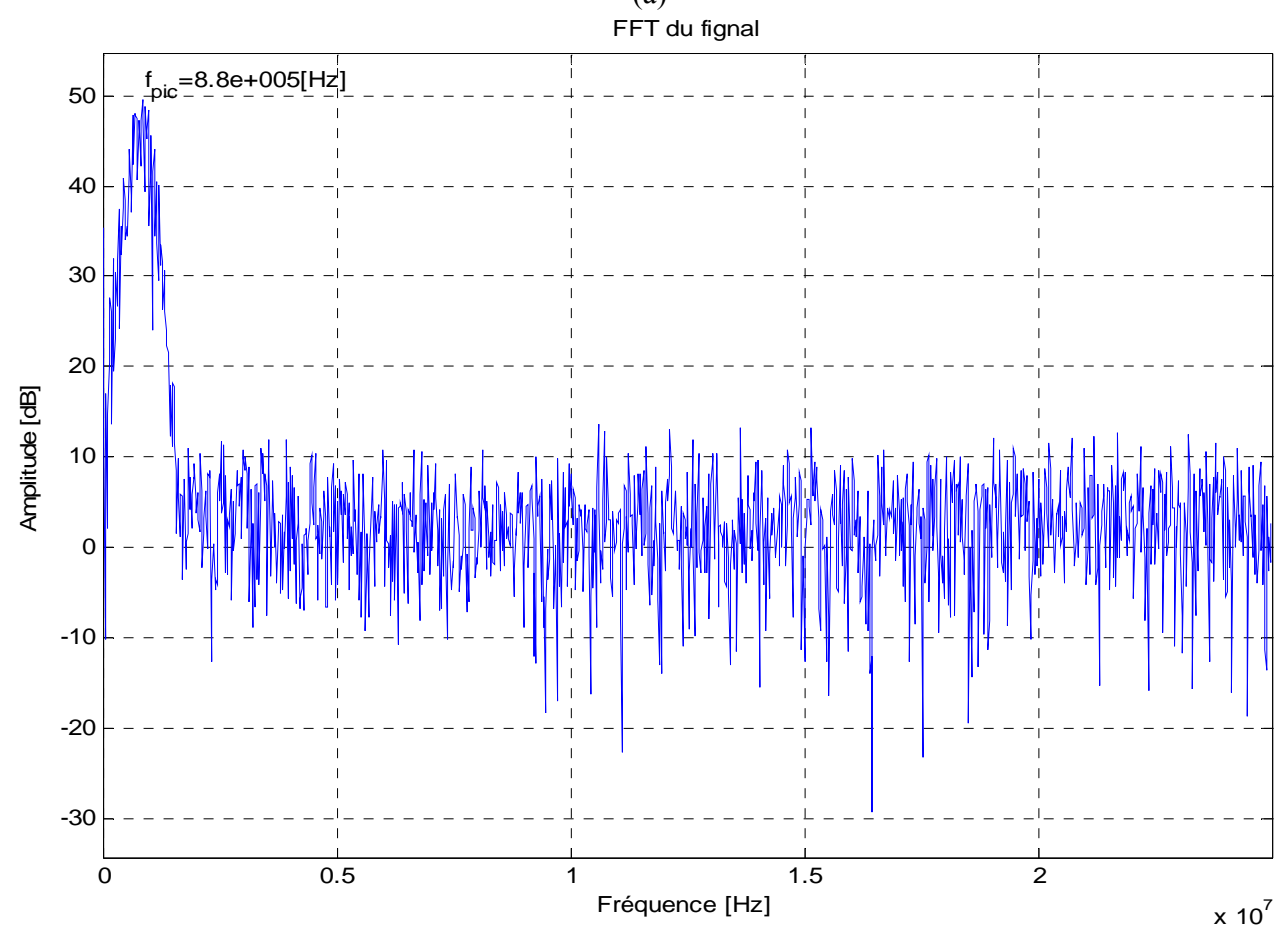

(b) 


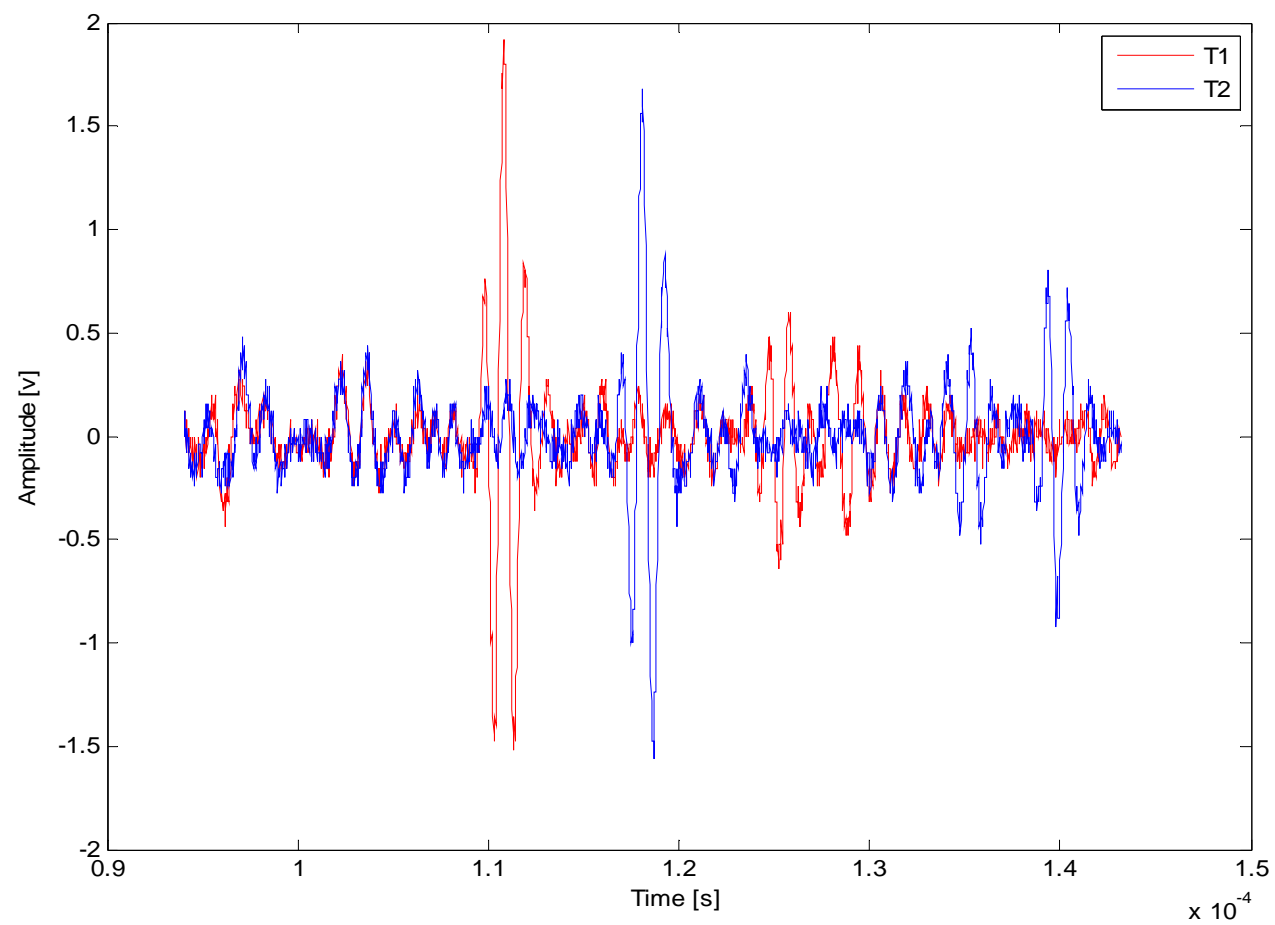

(c)

Fig. 6 a) Transient ultrasound waves; b) Fourier transform; Changes in signal wave form during setting.

Absolute Values of Ca,b Coefficients for $a=12345 \ldots$

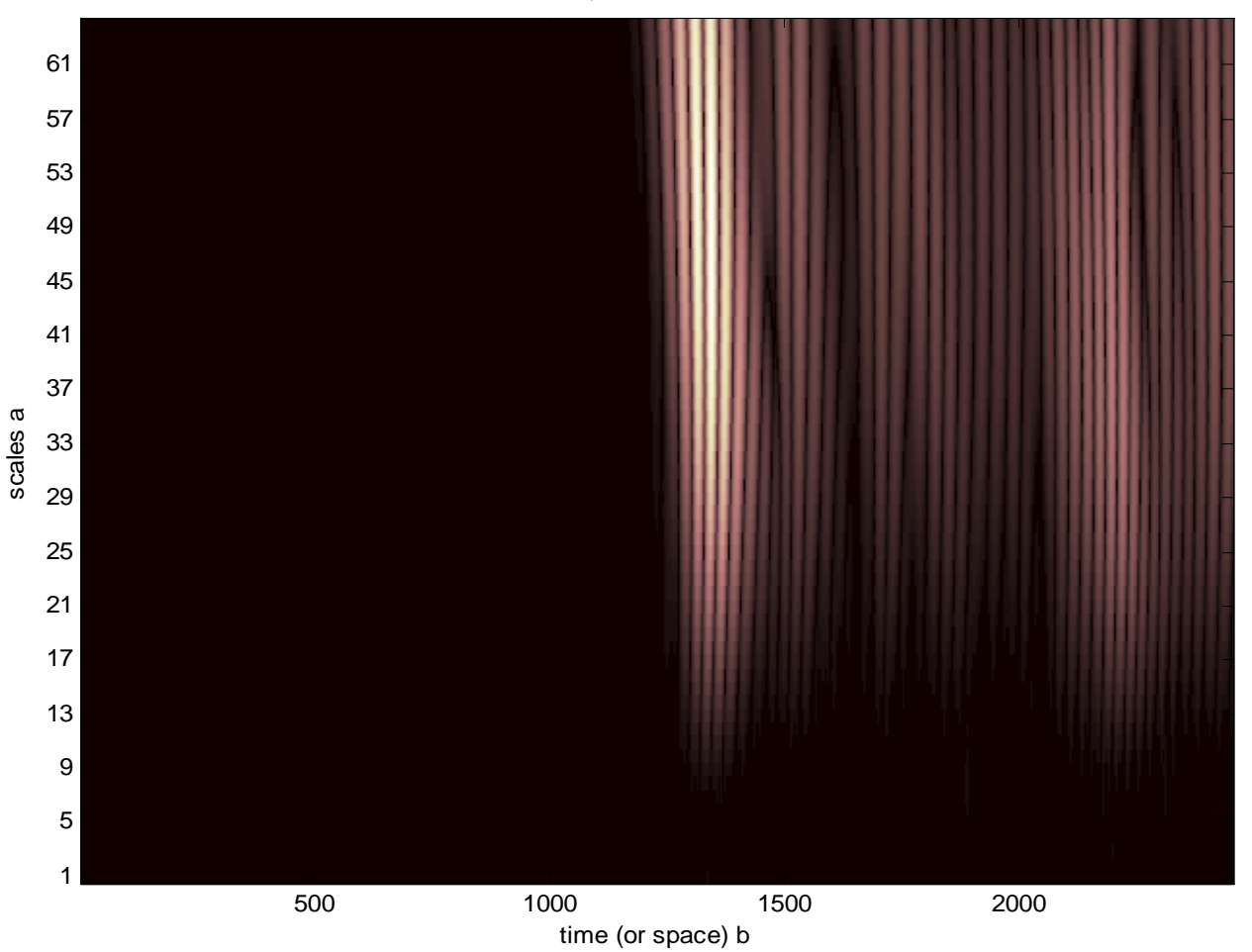

Fig. 7 wavelet transform of ultrasound wave. 
Table1 Ultrasound parameters for different ratio $\mathrm{E} / \mathrm{C}$.

\begin{tabular}{|c|c|c|c|c|c|c|c|c|c|c|c|c|c|}
\hline Parameters & $\mathrm{E} / \mathrm{C}$ & 1 & 2 & 3 & 4 & 5 & 6 & 7 & 8 & 9 & 10 & 11 & 12 \\
\hline \multirow{2}{*}{$\mathrm{t}_{\mathrm{v}}(\mu \mathrm{s})$} & 0.3 & 35 & 33 & 30 & 27 & 26 & 24 & 20 & 19 & 18 & 16 & 13 & 10 \\
\hline & 0.6 & 40 & 38 & 37 & 35 & 32 & 30 & 28 & 24 & 21 & 19 & 17 & 14 \\
\hline \multirow{2}{*}{$\mathrm{Vp}(\mathrm{m} / \mathrm{s})$} & 0.3 & 857.1 & 909.1 & 1,000 & $1,111.1$ & $1,153.8$ & 1,250 & 1,500 & $1,578.9$ & 1,666 & 1,875 & $2,307.7$ & 3,000 \\
\hline & 0.6 & 750 & 789.5 & 810.8 & 857.1 & 937.5 & 1,000 & $1,071.4$ & 1,250 & $1,428.6$ & $1,578.9$ & $1,764.7$ & $2,142.9$ \\
\hline \multirow{2}{*}{ E (Gp) } & 0.3 & 3.5 & 3.1 & 4.2 & 5.2 & 5.6 & 6.6 & 9.5 & 10.5 & 11.7 & 14.8 & 22.4 & 37.9 \\
\hline & 0.6 & 2.4 & 2.6 & 2.8 & 3.1 & 3.7 & 4.2 & 4.8 & 6.6 & 8.6 & 10.5 & 13.1 & 19.3 \\
\hline
\end{tabular}

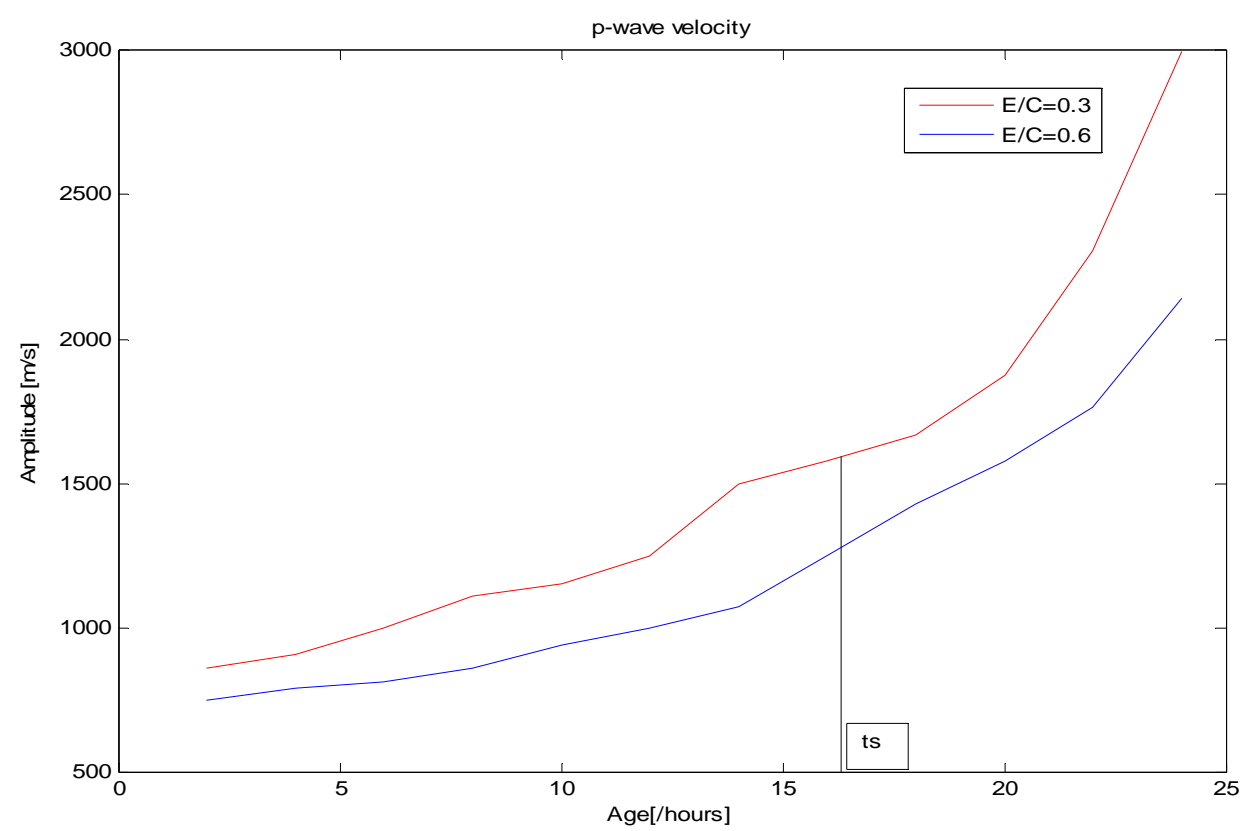

Fig. 8 Evolution of compression wave velocity over time.

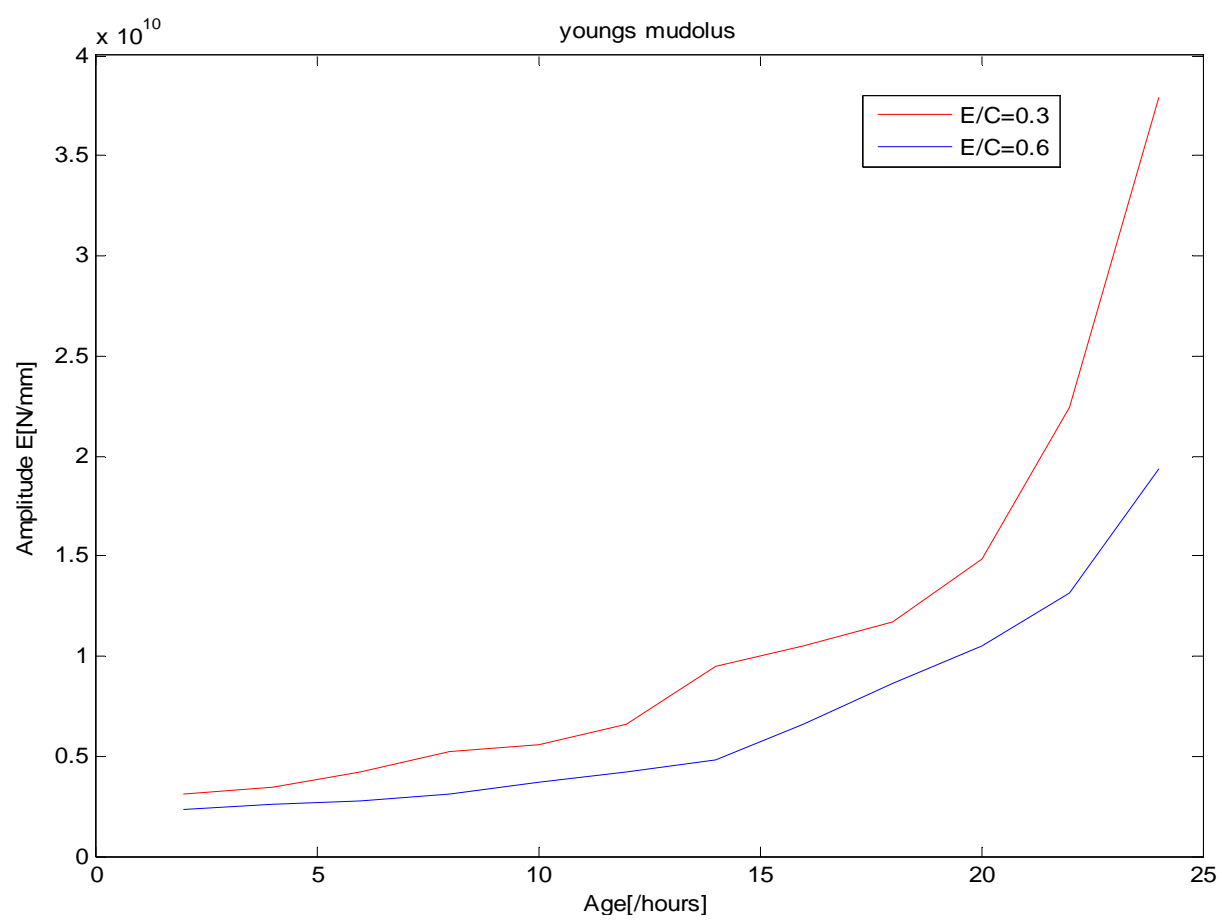

Fig. 9 Young's modulus versus age. 


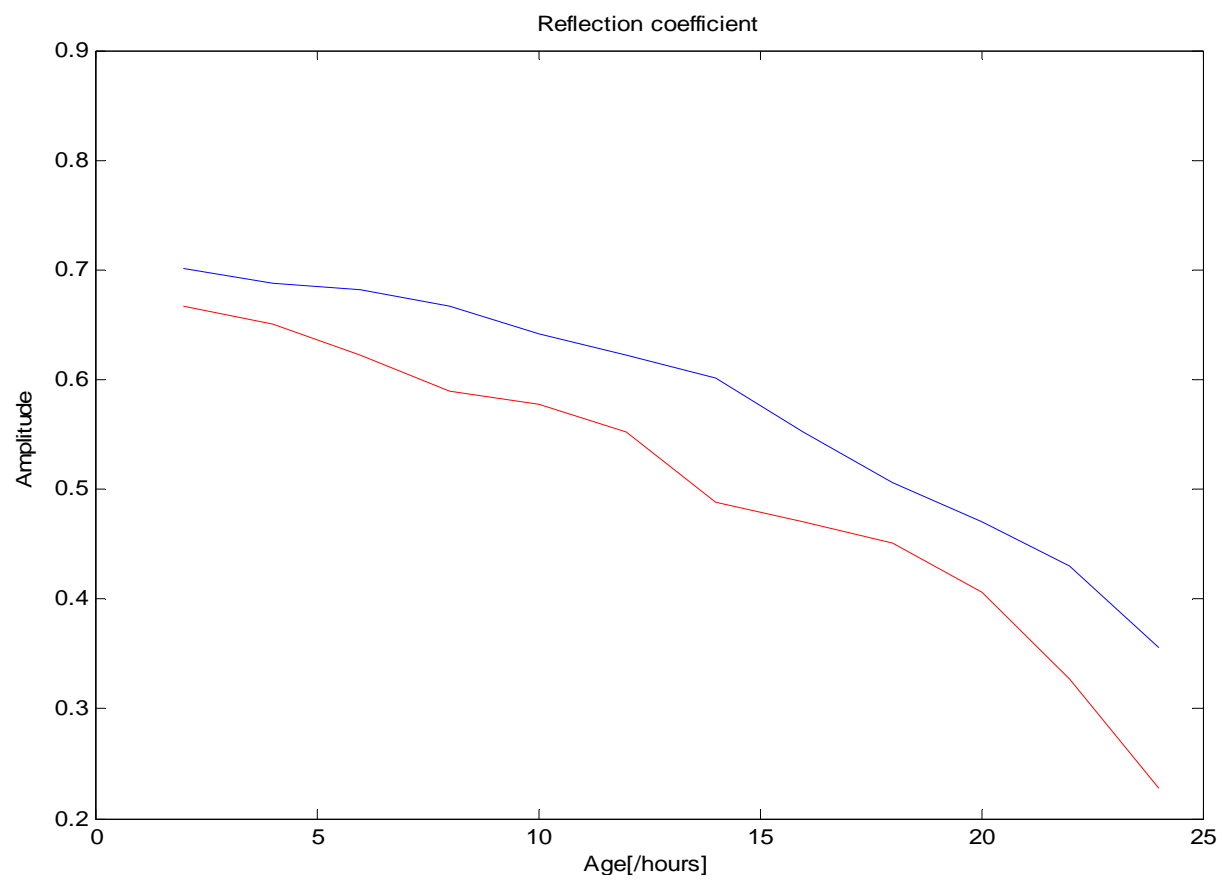

Fig. 10 Reflection coefficient versus age.

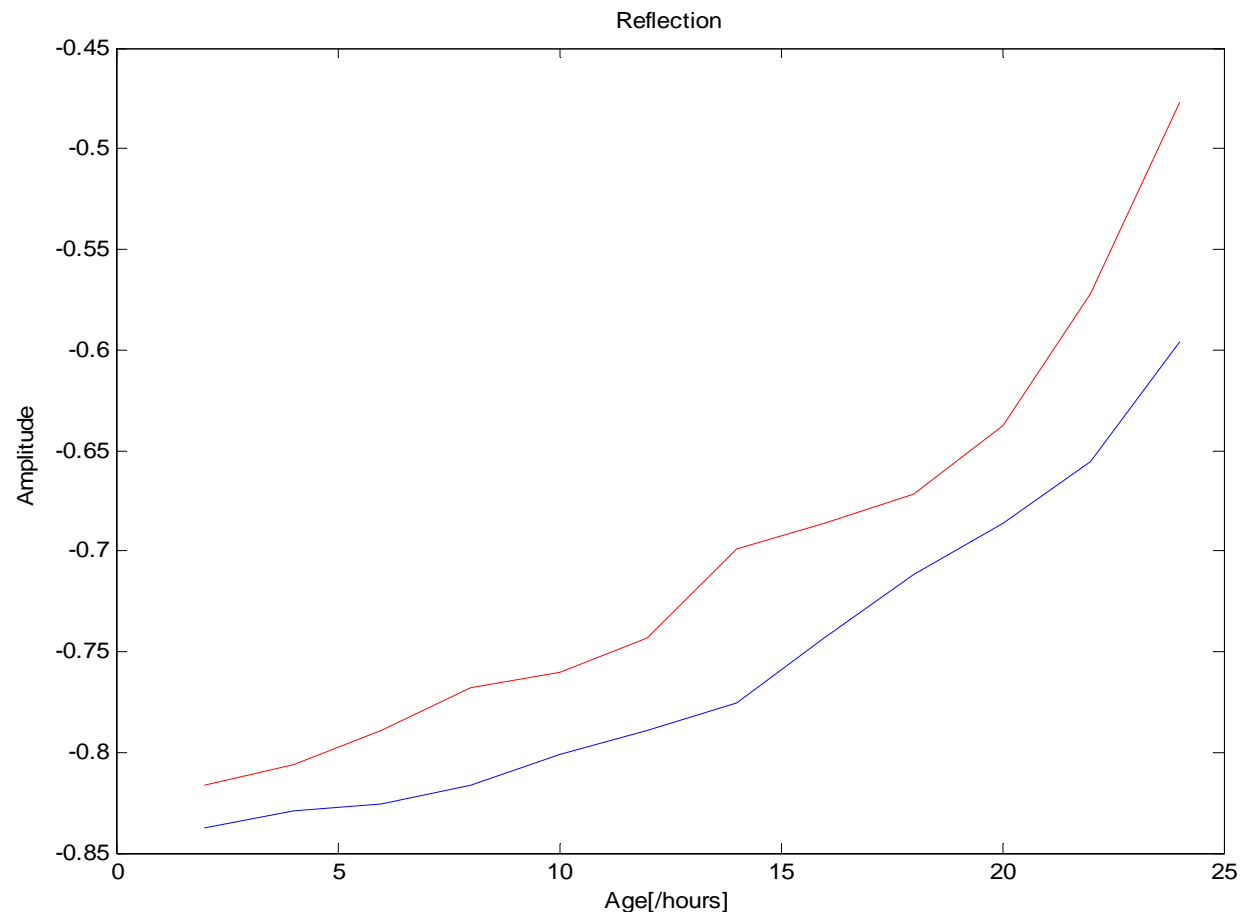

Fig. 11 Reflection versus age.

\section{Discussion of Results}

Ultrasonic pulse velocity development can be used to describe the micro structural changes in the cement paste caused by hydration process. In the first stage, ultrasonic pulses transmitted through a fresh cement paste are not recorded by measuring system, probably because of a large attenuation of the paste. After approximately 1.5 hours ultrasonic pulses are being recorded. 
Initial upv is at approximately $500 \mathrm{~m} / \mathrm{s}$. The reason of this low initial velocity is a large amount of very small air bubbles entrapped in the cement paste during mixing. upv has a constant increase up to about 1,500 $\mathrm{m} / \mathrm{s}$ which is attributed to the growth of ettringite crystals which fill out the capillary space and in that way reduce the distances between them. It was presented by several authors that at the speed of ultrasonic wave of $1,500 \mathrm{~m} / \mathrm{s}$ cement paste, with w/c ratios varying from 0.3 to 0.6 transforms from a liquid to a solid state. Setting process is finished and a hardening process starts. The setting process of cement paste is caused by the increase in the connectivity of the cement particles. With the progress of hydration, connectivity increases and this causes increases of the upv. At the same time water from capillary pores is constructed in the chemical reactions with cement and capillary spaces become partially filled with hydration products.

\section{Conclusion}

The results obtained in this paper show that the prism-technique could be used for evaluating the setting and hardening of concrete.

Compression wave velocity was monitored over a 24 hours period and plotted results show that the setting time (ts) could be defined easily from the slope of the curve. The rate of change of reflection coefficient could also be used in the same way. The convenient concrete mixtures could be selected for different practical applications. The future work will be concentrated on the exact definition of ts.

\section{References}

[1] Schubert, F., and Bouhadjera, A. 2011. "Apparatus for the Non-destructive Testing of Samples Using Ultrasound Waves." Fraunhofer-Gesellschaft zur foerderung der angewan forschung E. V., US patent application 20110179873, 28 Jul.

[2] Schubert, F., and Bouhadjera, A. 2013. "Apparatus for the Non-destructive Testing of samples Using Ultrasound Waves." Fraunhofer-Gesellschaft zur foerderung der angewan forschung E. V., Us patent 8468889, 25 Jun.

[3] Bouhadjera, A. 2013. "Dispositif Pour Tester des échantillons sans les détruire au moyen d'onde ultrasonores." Fraunhofer-Gesellschaft zur foerderung der angewan forschung E. V, Ep patent 2326948B1, 2 janvier.

[4] Bouhadjera, A., and Bouzrira, C. 2005. "High-frequency Ultrasonic Testing of Young Cement Based Materials Using the Prism Technique." ndt \& E international 38: 135-42.

[5] Carino, N. J. 2001. "The Impact-Echo Method and Overview." Building and Fire Research Laboratory, National Institute of Standards and Technology, Proceeding of Structures Congress and Exposition, May, USA.

[6] Christian, U. G., Hans, W. R., and Alexader, T. H. 2005. "Ultrasound in Through-Transmission." Advanced Testing of Cement-Based Materials during Setting and Hardening, Rilem TC 185-ATC: Final report, September.

[7] Matias, K., Karim, H., and Ferdinand, S. R. 2005. "Determination of Mechanical Properties of Concrete at very Early Ages Using Advanced ndt Techniques." Advanced testing of cement-based materials during setting and hardening, Rilem TC 185-ATC: Final report, September.

[8] Nicolas, R., and $\mathrm{Nel}$ de belie. 2009. "Monitoring the Setting of Concrete by Measuring the Change in Ultrasonic P-Wave Energy." NDTCE'09, Non-Destructive Testing in Civil Engineering, Nantes, France, June 30th. 